\title{
A strengths-based approach to mentoring women entrepreneurs: how to free the strengths within them
}

\author{
Olga Stavropoulou ${ }^{1,2,3,4}$ and Sophia Protopapa ${ }^{2,5^{*}}$
}

\author{
* Correspondence: \\ protopapa@knowl.gr \\ ${ }^{2}$ Business Mentors, Greek Part of the \\ European Network of Mentors for \\ Women Entrepreneurs, 335 \\ Acharnon Str., 11145 Athens, Greece \\ ${ }^{5}$ Knowl, Social Enterprise for \\ Education and Lifelong Learning, 10 \\ Iliopoulou Str., 11145 Athens, Greece \\ Full list of author information is \\ available at the end of the article
}

\begin{abstract}
Entrepreneurship has been considered the route towards European recovery and growth. The contribution of female entrepreneurship is of utmost importance as women bring into business ventures attributes such as team spirit, synergy, intuition, multi-tasking, and social awareness, beneficial both for entrepreneurial advancement and society. However, women wishing to pursue or/and maintain a successful entrepreneurial path are still faced with a gender gap prevailing in entrepreneurial practice and culture. Despite an encouraging recent increase in the number of women entrepreneurs, their representation is still limited to about 30\% of EU entrepreneurs. Mentoring is considered effective for personal and professional development of women entrepreneurs. It is realistic and adjustable to their true needs, expectations and business context. In a mentoring relationship of equality and trust, the mentor provides just-in-time knowledge and experience which support the woman entrepreneur in making decisions. The mentee learns from the mentor's experience and is more likely to transfer new learning on her business because it is more meaningful and necessary. Strengths-based mentoring can be a more effective and dynamic developmental process for women entrepreneurs. It enhances social interaction in which the mentor facilitates the mentee to discover her strengths and use them to achieve her goals. Being aware of her strengths, the mentee engages in self-reflection and evaluation and develops both personally and professionally. The mentor is instrumental in enabling her to discover her strengths, use them in her business and release her potential. This paper presents an innovative strengths-based approach, 'feel the mentoring ${ }^{\mathbb{O} !}$, a human-oriented model that enhances personal experience and the professional milieu in which a woman entrepreneur performs. The feel the mentoring ${ }^{\circ}$ enables the mentee to discover, recognise, emerge, free, use, combine and evaluate her strengths and sustainably employ them in her activities. The practical value of this approach is discussed through the real case of a woman entrepreneur who experienced strengths-based mentoring. While herein discussed in the context of female entrepreneurship, feel the mentoring ${ }^{\circ}$ is not restrained to it. It can be successfully applied with male entrepreneurs, employees or unemployed individuals who wish to gear their inner strengths to professional and personal advantage.
\end{abstract}

Keywords: Female entrepreneurship, Natural talents, Strengths, Strengths-based mentoring, feel the mentoring ${ }^{\odot}$

\section{Springer}

(c) 2013 Stavropoulou and Protopapa; licensee Springer. This is an Open Access article distributed under the terms of the Creative Commons Attribution License (http://creativecommons.org/licenses/by/2.0), which permits unrestricted use, distribution, and reproduction in any medium, provided the original work is properly cited. 


\section{Review}

\section{More women entrepreneurs are beneficial to the European economy}

Entrepreneurship has long been suggested as a 'must-go-path' towards European recovery and growth, especially during the last years when the entire continent has been faced with a severe economic crisis. In this context, the contribution of female entrepreneurship is of utmost importance especially as women bring into the business practice attributes such as team spirit, synergy, intuition, multi-tasking, commitment and social awareness that are beneficial both for entrepreneurial advancement and for society as a whole. However, women wishing to pursue or/and maintain a successful entrepreneurial path are still faced with a persistent gender gap prevailing in both entrepreneurial practice and culture. Despite an encouraging recent increase in the number of women entrepreneurs, their representation is still limited, amounting to only about $30 \%$ of EU entrepreneurs. In fact, 'Not only is this gender imbalance unfair to women, but it is also damaging to the European economy at a time when the EU needs more entrepreneurs to fuel its economic recovery and create the SMEs which are the backbone of the European economy and its main job-creation engine' ${ }^{\text {a }}$.

\section{A strengths approach for the 'business weak' feminine gender}

Women entrepreneurs still constitute a minority in the business world mainly due to various barriers that arise from their feminine gender such as anachronistic societal and cultural perceptions, plans to make a family while in business, need to undertake multiple roles in their business and at home, the difficulty to convince investors and raise capital, feelings of insecurity and fear of failure in a tough business world (Winn 2005). In order to overcome these barriers, there have been numerous (formal and informal) approaches, initiatives, programmes, methodologies and tools that actually help women to 'surf' successfully through the waves of the business growth cycle, making the constraints they are faced with irrelevant.

As presented in this paper, one promising and dynamic approach for any woman entrepreneur or wannabe entrepreneur relies on her efforts to identify, understand and make proper use of her existing inner strengths in her business endeavours. During this process that actually sets free the strengths within her, a mentor is catalytic in facilitating her to recognise her strengths, with a focus on those that empower her business growth, and at the same time manage to surface and exploit her untapped potential. It is noted that this approach fits both sexes and it is practiced by both; nevertheless, women tend to be more receptive to trying such informal methods.

\section{Bringing 'can dos' on stage}

Strengths-based mentoring is a leading-edge approach that yields positive results as it is an evolutionary experience for both mentors and mentees. On the one hand, the mentor introduces the mentee, in this case a woman entrepreneur, to this positive psychology perspective that enables her to recognise, identify, understand and build on her strengths so as to apply them in her different roles and phases of business growth. On the other hand, the mentor himself/herself, through this evolutionary interaction, further deepens his/her knowledge and awareness on how to surface, combine, exploit and use their own strengths while in parallel, this 'extra wisdom' accelerates their personal and professional growth. 
In general, strengths-based mentoring focuses on individual virtues, talents and human ability for fulfilment rather than on weaknesses and deficits. It is a positive perspective that elaborates on, and culminates in, the things one 'can do' rather on those that one cannot. The logic is to put less effort and get more energy from those inner strengths that accumulate toward one's evolution and make a difference in one's growth path, rather than waste most energy on things that could only improve to a pre-defined and limited scale, preventing growth acceleration (Linley 2008). This dynamic approach achieves to manifest what one can do thus creating also a positive chain reaction to the immediate working (and social) environment such as colleagues, workforce and the business culture as a whole (He 2009; Sullivan 2000).

\section{Starting with a trustworthy relation between mentor-mentee}

A caring relationship between the mentor and the woman entrepreneur is paramount for facilitating the latter to develop her inner strengths while enabling her to elevate from average to A+ performance (Linley 2008). Strengths-based mentoring can prove an empowering experience and a meaningful drive for the woman entrepreneur. It can illuminate the path to achieving her true potential and maximum performance out of the cultivation of mutual respect and an honest rapport between herself and her mentor, openness and trust as well as encouragement and interest by the mentor to genuinely listen, understand and respond to her concerns. Such an experience can provide a fertile ground and an ample opportunity for the woman entrepreneur to speak to her mentor about the strengths she is already putting to use and with what results, the strengths she wants to develop further and use more and about her intended strategy for achieving these towards goal realisation. By providing constructive feedback and continuous support, the mentor enables her to make the most out of her strengths in her professional and personal life while encouraging and inspiring her to untap her potential (Clifton et al. 2006; St. Haines 2003).

\section{We all have talents}

Aiming to provide a deeper insight into strengths-based mentoring and unravel the ways in which it can be applied in the context of female entrepreneurship, the meaning and importance of talent are primarily discussed given that it constitutes the basis of strengths. 'A talent is a naturally recurring pattern of thought, feeling, or behavior that can be productively applied' (Clifton et al. 2006, p. 2). Talents exist naturally and are authentic aspects of individual uniqueness. They correspond to the innate ability that people have to combine them, deliver performance and achieve goals, in processes which are directly connected. Individuals who utilise their talents increase the likelihood of fulfilling their potential, providing effective performance on recurring occasions and situations, while achieving not good but excellent performance at work and all areas of social activity. This constitutes solid justification as to why individuals should aspire to identify, understand and cultivate their innate talents (Clifton et al. 2006; Clifton and Harter 2003).

Natural talents are elevated to strengths through refinement enabled by the formal or informal acquisition of knowledge, skill and experience enhanced by practice. This implies the risk that talents might be wasted in case they are not further cultivated. Strengths capacitate the individual to consistently provide dynamic and excellent performance in a given task (Clifton et al. 2006; Clifton and Harter 2003). 


\section{We all have strengths}

'A strength is a pre-existing capacity for a particular way of behaving, thinking or feeling that is authentic and energising to the user, and enables optimal functioning, development and performance' (Linley 2008, p. 9). Strengths are, indeed, an integral part of our human identity, constituting our intrinsic and inherent qualities that define us as individuals. However, only about one third of us are conscious and appreciative of them or certain about how we can best put them to use to achieve desired outcomes. This causes our inner treasure and the exciting opportunities lying ahead of us for a fulfilling life to perish.

A strength is delineated in three elements - performance, energy and use-all of which must be present in order for strengths to underpin and sustain optimal performance. Using strengths energises and motivates individuals to contribute discretionary effort towards attaining their goals while it nourishes in them a feeling of achievement due to the fact that they perform in accordance with the things they are best at. Research indicates that when individuals use their strengths, they feel happier, more confident and proud of themselves and more engaged at work, and they perform better while experiencing a sense of resilience for achieving their goals. To produce these outcomes, however, strengths have to be used frequently (Linley et al. 2010a, 2010b; Linley 2008).

Research by Linley et al. (2010a, 2010b) resulted in the identification of numerous individual strengths and the compilation of the major ones in five strengths families: (1) 'Being', the first of the strengths families, represents the way of being in the world and comprises strengths such as authenticity, courage, curiosity, humility, legacy, mission, personal responsibility, pride and un-conditionality (2). The utilisation of 'communicating' strengths is conducive to the way of giving and receiving information. This family encompasses strengths such as explainer, feedback, humour, listener and narrator (3). The strengths family of 'motivating' explains what drives individuals toward action comprising among others adventure, bounceback, change agent, competitive, drive, growth, improver, persistence (4). The 'relating' strengths family underpins the way an individual relates to others and consists of attributes such as connector, emotional awareness, enabler, esteem builder, persuasion, and relationship deepener (5). Last, the 'thinking' strengths family highlights what individuals pay attention to and how they approach situations including adherence, detail, incubator, innovation, judgement, planful, prevention, reconfiguration, and strategic awareness among others.

\section{'feel the mentoring ${ }^{\odot}$ : a strength-based approach to mentoring}

The feel the mentoring ${ }^{\odot}$ strengths-based approach is a human-oriented model that enhances personal experience and the professional milieu in which individuals interact. It fosters positive attitude change that results from a process of discovery, recognition, emergence, freedom, use, combination and evaluation of strengths that inevitably leads to better performance, productivity and well-being, which are the essential ingredients for successful personal and business experience and growth. The feel the mentoring ${ }^{\odot}$ is a dynamic strengths-based mentoring model with the main goal to free the strengths individuals have within themselves, and the strengths not only of the mentee but also of the mentor himself/herself, in a process of mutual development for both parties involved.

The feel the mentoring $\odot$ guides the woman entrepreneur towards a personal and unique pursuit with the aim for her to untap her potential and achieve her goals. The 
basic stages of the model focus on her uniqueness and strengths, rather than on her weaknesses, and on the ways in which she can combine them effectively and utilise them successfully.

\section{Stages to free the strengths within you}

The discovery, recognition, emergence, freedom, use, combination and evaluation of strengths underpin the personal and unique pursuit and development of one's inherent talents which enable the realisation of one's potential and goal achievement (Figure 1). These are, in fact, the main steps of the feel the mentoring® model (www.feelthementoring. gr) that focuses on the assets of individuals as well as of groups, as opposed to their weaknesses, and on the ways in which these assets can be productively combined and employed to sustain success at all levels.

Discovery. This initial stage is crucial in the mentoring relationship and one which instigates the strengths-based approach. The mentor, already trained in the strengths based approach and aware of his/her strengths spotting skills, facilitates the mentee in discovering her own strengths, through the use of a scientific test and powerful questions, with the aim to raise her awareness of her strong self. This stage is of paramount importance for the mentee since it can assist her in embarking on a journey of self-reflection, a process of understanding and evaluating herself, which raises her awareness of her inner strengths and the possible ways to combine and apply them in her activities and achieve her goals.

Recognition. Through debriefing on the test results and discussion, the mentor assists the mentee in thinking of ways in which she is already putting her strengths in use in her everyday activities or reflecting how she used them successfully in the past. The mentee recognises the activities in which she feels capable and is effective, the things she enjoys doing every day, the activities that make a day really good for her, her achievements to date and the situations in which she experiences flow, authenticity and positive energy. At this stage, she also recognises her weaknesses in activities in which she does not deliver optimal performance. These are activities which she must not disregard but rather control or change to prevent them from interfering with her daily work.

Emergence. This stage complements the preceding ones, being future oriented and core in strengths-based mentoring. It cultivates the ground on which the mentee will prepare to accept and free her strengths. Apart from asking the mentee to describe experiences in the present or recall past ones in which she used her strengths, the mentor also guides her to

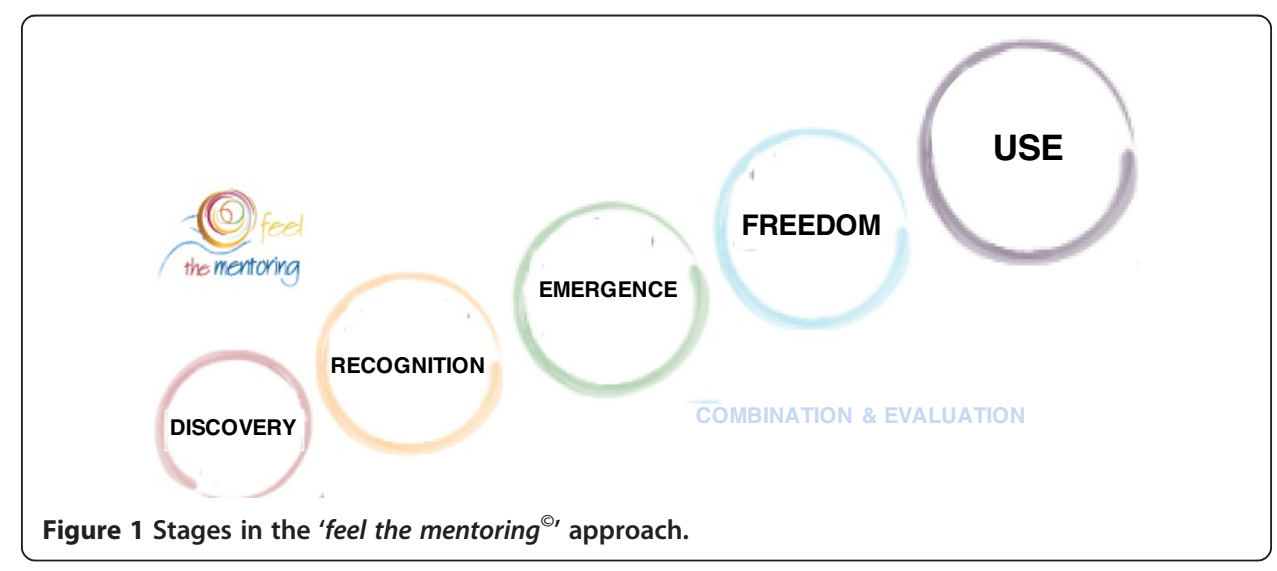


think of ways in which she can use her strengths in the future by encouraging her to see into her goals and expectations, her vision for her life and her business and her aspirations about what she wants to achieve. In this process, she can realise how she can further develop and refine her strengths in order to use them in novel situations and ways and how she can control her weaknesses. This stage could be faced with resistance on the part of the mentee arising from existing habits, fears, weaknesses and social and cultural perceptions, or from difficulty in realising how the emerging strengths can contribute to the attainment of goals.

Freedom. During this reviving stage, the mentee manages resistance, having reached a high level of understanding of the potential and dynamics of her strengths. Having a better picture of her strong self, she experiences a sense of fulfilment, personal satisfaction and optimism that her goals can be accomplished by putting her strengths in action. The mentor guides her through the steps of goal setting and action planning, and she realises how she can use and combine her strengths to achieve success. This stage is, in fact, one of personal transformation which enables the mentee to perceive ways in which she can change and respond to challenges in a more positive and effective way, engage and commit to action and identify measurable results she wishes to achieve by using her strengths.

Sustainable and optimal use and combination of strengths. Following the previous stages, the mentee is now putting her strengths into use in a meaningful and systematic way in order to achieve her goals. Appropriately using and combining her strengths enables her to anticipate and manage challenges, identify options and their consequences, select the best option for a given situation and context and deliver optimal performance. This stage implies, and requires, that the mentee is flexible and proactive in putting her diverse strengths to the best of use each time while taking caution to avoid overusing them. She is also aware of the value of cooperating with others and combining, or complementing, her own strengths with those of colleagues.

Evaluation. Evaluation is, in essence, a continuous and integral process of strengthsbased mentoring. The mentor provides constructive strengths-based feedback which focuses on how the mentee uses her strengths and recommends ways in which she can employ them in similar or novel situations and contexts. Through discussing the mentee's performance, the mentor facilitates her in evaluating whether she uses her strengths sufficiently each time, and with what results, since overusing them could produce unwanted outcomes or affect future possibilities. This approach creates opportunities for the mentee to further develop her strengths and adopt fresh insights into professional and personal prospects. By providing strengths-based feedback, the mentor further fosters the mentee's engagement and commitment, contributing to higher performance and achievements in her business.

\section{feel the mentoring ${ }^{\odot}$ in action}

This section of the paper presents a successful real case study of a woman entrepreneur who applied the feel the mentoring ${ }^{\odot}$ stages, i.e. discovery, recognition, emergence, freedom, use, combination and evaluation of strengths, to optimise business performance. It is noted that feel the mentoring ${ }^{\odot}$ recommends the use - at the initial mentoring phase - of a strengths assessment and development tool, such as Realise $4 \mathrm{M}$ model ${ }^{\circ}$, as it can provide some initial insights that could prove useful to the subsequent mentoring phases. Actually, the case study that follows refers to a woman entrepreneur who is over 40 years old, a 
married mother, who has been successfully running and growing her business, a consultancy firm, regardless of the severe economic crisis that Greece is faced with. The woman entrepreneur completed the Realise 2 strengths test which yielded meaningful and interesting results also considered in the mentoring sessions that followed. The aim of this real case study is to demonstrate, in practical terms, the ways in which strengths-based mentoring can support and enable a woman entrepreneur to achieve her goals by discovering and exploiting her own inner strengths as well as those of her team. It is noted that by adopting a strengths-based approach towards others, an entrepreneur can free his/her employees' strengths and elicit their discretionary effort and maximum performance, recruit, retain and engage individuals best suited to the business culture and needs, and enhance business flexibility by investing on employee potential. A strengthsbased perspective can further support and enhance teamwork by focusing on the team's strengths simultaneously to the allocation of tasks according to individual strengths; set the ground for dealing with change by enabling individuals to think and act in new and creative ways; and enhance employee job satisfaction and self-fulfilment at work by increasing their opportunities to do not only the job they are good at but also the job they love (Linley 2008; Pegg and Moore 2005).

The case study of the woman entrepreneur is presented as follows:

The woman entrepreneur (mentee) was work-overloaded having to constantly better the end results of her team of employees, thus her daily business was over-burdened and prolonged as she had to undertake extra reading, analysis, evaluation and thus more work time in order to maintain high-quality levels of business outcomes and client satisfaction. As a result, her team of employees relied more and more on her than on themselves to minimise errors, deepen knowledge, undertake responsibility for the final outcome, etc., while she was obliged to work late hours to cover the heavy work overload (less personal time, tiredness, frustration, concern for two-speed performance); and most importantly, she was prevented from focusing on the strategic development of the business.

After having completed a strengths assessment test, during the initial mentoring session, she identified one of her top strengths to be her effectiveness in decision making, namely, that she is good at it (based on her previous proven record, performance and business results) and she feels at her best when she practices it, i.e. with the process of comprehending a situation and having to take a decision under any circumstance. In brief, she enjoys decision making, she gets energised when she practises it, and in the majority of times she takes the right decisions.

She realised that her strength in decision-making could be the solution to the challenge she was being faced with, as long as she took the time to review the challenge, analyse the situation, and be determined to resolve it. Interesting enough, she admitted in taking numerous decisions during her daily business, and to be good at it; however, she did not think of adopting the same procedure to address the challenge that de-energised her on a personal business/personal level. Following an intense strengths-based mentoring session, she concluded that she simply had to make time in her calendar to deal with her personal business challenge as she would do with any other project, deal or initiative that seemed more tangible. As such, she set her personal entrepreneurial goal: to better her business performance by working on her personal challenge. 
The emergence of her decision-making strength, but in a specific goal-setting context that touched her on a more personal and sentimental level, led to the freedom and use of her inner strength while combining/complementing with other strengths such as her good judgment, analytical thinking, and commitment to decision making that facilitated her to modify the way work had been carried out until that time so that the employees would implement delegated tasks and responsibilities at high-quality levels without needing her close and continuous assistance and supervision. To this aim, she decided to alter first the way she worked and further to guide her employees and enable them to undertake full responsibility of their work and their deliverables to the customer. She had to combine other strengths she recognised during the mentoring sessions in order to let go of and counterbalance other concerns for the end results (e.g. concern if the end result is not of high quality how it would affect the company reputation, profit and development). For example, being strong in helping others grow, in transmissibility, in persuasion and motivational skills could help her explain why and how this new work model is beneficial to all, and the business itself, and help her team understand the importance of the exercise and at the same time engage them in this new working model.

Moreover, her mentor suggested reviewing the results of the strengths test that the woman entrepreneur had taken in parallel with the mentoring sessions. Thus, according to the Realise2@ results and debrief, she was attributed with strengths such as Adherence, Catalyst and Feedback. These strengths were demonstrated in activities which she performed frequently and delivered high-quality work while also gave her energy. She understood that she could use her Adherence strength so as to focus on her goal and achieve it and also her Catalyst strength since she wanted to introduce and implement change as to the way her employees, and herself, worked. Further, she combined and complemented these strengths with her Feedback strength which could help her debrief her employees on their performance. According to the same test results, the behaviours she was frequently exhibiting at that period, at which she performed well but did not give her energy, were Optimism, Efficacy and Persistence. In fact, she felt that she was overusing her Efficacy and Persistence strengths in order to deliver high performance, often to compensate for the employees' lower performance, and this was one of the main reasons as to why she felt de-energised. Further, she admitted that if she continued overusing them she would experience burn out. Thus, she had to act fast by making an effort to control these strengths, in particular, Efficacy and Persistence while simultaneously empowering her employees to undertake responsibility for their work. In this case, too, she used the Catalyst and Feedback strengths to compensate for her Efficacy and Persistence behaviours and introduce new ways of working in the company. The fact that Strategic Awareness was identified as a weakness (activities at which she did not deliver high performance and which de-energised her as well), could be justified since, being concerned with the everyday performance of the employees and the deliverables they had to produce, most of her time and attention was absorbed away from business strategy. Finally, the strengths that she was not employing at that period and which she could employ more in the future in order to reach her goal were Explainer, Narrator, Emotional Awareness, Order, Detail, Mission, and Planful. She truly believed that using these strengths would primarily 
motivate her since she would perform well and would also draw energy by applying them. Further, they could help her achieve her goal of introducing change in the ways her employees worked. In particular, she felt that using the strengths of Explainer, Mission and Planful more would enable her to communicate better with her employees, explain her feelings and subsequently draft a plan based on common understanding so as to change the way both her and the employees worked.

When she was asked what was the first action she took, following the mentoring session, she said that she organised bilateral meetings with all her employees during which they discussed their performance and gave her the opportunity to hear their own perception and opinion about their work and outcomes and about how new ways of working could be introduced. In these meetings, she also admitted her own feelings of being exhausted and de-energised since she had to supervise and complete their work on a continuous basis. Following this initial action, she then drafted a detailed action plan on how to move forward in order to optimise performance, by re-engineering task delegation. The action plan also included the attendance of specialised strengths-based training by the employees since she regarded it as essential for facilitating the employees to discover their own strengths while she would subsequently be able to debrief them on how they employed them at work.

Four months later, important positive results are already recorded, although the goal setting period is set for six months. Still, current progress and findings are impressive and encouraging so as to continue effort on applying the strengths approach model in meeting the challenge and reaching the goal set. So far, higher staff productivity is reported as well as better daily work schedule for the woman entrepreneur (although still working long hours) which has allowed more time available for strategic orientation and business growth. Furthermore, by adopting the principles of strengths-based mentoring herself, she now feels better in helping her team to discover and free their inner strengths, which increases their work performance, satisfaction and experience.

\section{Conclusions}

\section{A positive outlook for the future}

As the case study above suggests, strengths based mentoring can prove a solid base in order for the woman entrepreneur to be able to develop her self-confidence and selfefficacy as well as a clear view of how she can best respond to the business environment through self-reflection and evaluation based on her strengths (Schwille 2008; Clutterbuck and Lane 2004).

By adopting the strengths based approach, the woman entrepreneur had the opportunity to use her strengths and also help her employees free their own strengths. Their performance improved since they were able to untap their potential.

The strengths based approach can support and increase team performance by focusing on the members' strengths and by delegating tasks based on these strengths. This can prepare the ground for change by supporting employees to work in new and creative ways, and increase their job satisfaction and fulfilment by doing well a job they love (Linley 2008; Pegg and Moore 2005).

This positive experience involves the mentor as a genuine supporter. The mentor can help the woman entrepreneur to free her strengths through self-awareness, communication and feedback, authentic interest for her development and commitment to the mentoring 
relationship. The mentor's strengths as well as his/her experience need to be utilised to help the woman entrepreneur achieve her personal and professional goals though strengths' identification and creative use in her activities.

Overall, strengths based mentoring is a change in perception relating to the development of the woman entrepreneur. It is a positive attitude which shifts focus from survival to well being, from the existing situation to new opportunities, and from the effort to improve weaknesses to the capitalisation of strengths.

Finally, it should be noted that although this paper focused on strengths-based mentoring for women entrepreneurs, it is by no means restrained to this target group. In fact, strengths-based mentoring can be adjusted and applied in any formal mentoring relationship with women and men, entrepreneurs, employees or individuals who wish to discover, understand, refine and free their strengths in their work or personal life in order to exploit their untapped potential and deliver high performance on their way to success.

\title{
Endnote
}

${ }^{a}$ http://ec.europa.eu/enterprise/magazine/articles/smes-entrepreneurship/ article_10986_en.htm

Competing interests

The authors declare that they have no competing interests.

\section{Authors' contributions}

OS reviewed and edited the first manuscript and finalised it for submission. SP carried out initial research and produced the first manuscript of the article. Both authors read and approved the final manuscript.

\begin{abstract}
Author details
${ }^{1}$ Militos Emerging Technologies \& Services, 335 Acharnon Str., 11145 Athens, Greece. ²Business Mentors, Greek Part of the European Network of Mentors for Women Entrepreneurs, 335 Acharnon Str., 11145 Athens, Greece. ${ }^{3}$ Governing Committee of Women's Organisation of Managers and Entrepreneurs of the Hellenic Management Association (TOGME/EEDE), 200 lonias Ave. and lakovaton 61 Str., 11144 Athens, Greece. ${ }^{4}$ Hellenic Association of Young Entrepreneurs in Athens, Piraeus and Province, 9 Chavriou Str. and Praxitelous, 10562 Athens, Greece. ${ }^{5} \mathrm{Knowl}$, Social Enterprise for Education and Lifelong Learning, 10 lliopoulou Str., 11145 Athens, Greece.
\end{abstract}

Received: 15 February 2013 Accepted: 7 May 2013

Published: 13 June 2013

References

Clifton, DO, Andreson, E, \& Schreiner, L. (2006). StrenghtsQuest: discover and develop your strengths in academics, career and beyond (2nd Ed.). New York: Gallup.

Clifton, DO, \& Harter, JK. (2003). Strengths investment. In KS Cameron, JE Dutton, \& RE Quinn (Eds.), Positive organizational scholarship (pp. 111-121). San Francisco, CA: Berrett-Koehler.

Clutterbuck, D, \& Lane, G. (2004). The situational mentor: an international review of competences and capabilities in mentoring. Hants, UK: Gower Publishing Ltd.

St. Haines, T. (2003). The mentor-protégé relationship. American Journal of Pharmaceutical Education, 67(3), 458-464.

He, Y. (2009). Strength-based mentoring in pre-service teacher education: a literature review. Mentoring \& Tutoring: Partnership in Learning, 17(3), 263-275.

Linley, PA. (2008). From average to At: realising strengths in yourself and others. Coventry: CAPP.

Linley, PA, Garcea, N, Hill, J, Minhas, G, Trenier, E, \& Willars, J. (2010a). Strengthspotting in coaching: conceptualisation and development of the Strengthspotting Scale. International Coaching Psychology Review, 5(2), 165-176.

Linley, PA, Willars, J, \& Biswas-Diener, R. (2010b). The strengths book: be confident, be successful, and enjoy better relationships by realising the best of you. Coventry: CAPP.

Pegg, M, \& Moore, S. (2005). Strengths coaching in 90 minutes (p. 200). Gloucestershire: Management Books.

Schwille, SA. (2008). The professional practice of mentoring. American Journal of Education, 115, 139-167.

Sullivan, R. (2000). Entrepreneurial learning and mentoring. International Journal of Entrepreneurial Behavior \& Research, 6 (3), 160-175.

Winn, J. (2005). Women entrepreneurs: can we remove the barriers? International Entrepreneurship and Management Journal, 1, 381-397.

doi:10.1186/2192-5372-2-13

Cite this article as: Stavropoulou and Protopapa: A strengths-based approach to mentoring women

entrepreneurs: how to free the strengths within them. Journal of Innovation and Entrepreneurship 2013 2:13. 